\title{
A DESCRIPTION OF THE SOFTWARE ELEMENT OF THE NASA EME FLIGHT TESTS
}

\author{
Sandra V. Koppen \\ Lockheed Martin Engineering and Sciences Company \\ 144 Research Drive \\ Hampton, Virginia 23666 \\ USA
}

\begin{abstract}
In support of NASA's Fly-By-Light/Power-By-Wire (FBL/PBW) program, a series of flight tests were conducted by NASA Langley Research Center in February, 1995. The NASA Boeing 757 was flown past known RF transmitters to measure both external and internal radiated fields. The aircraft was instrumented with strategically located sensors for acquiring data on shielding effectiveness and internal coupling. The data are intended to support computational and statistical modeling codes used to predict internal field levels of an electromagnetic environment (EME) on aircraft.
\end{abstract}

The software was an integral part of the flight tests, as well as the data reduction process. The software, which provided flight test instrument control, data acquisition, and a user interface, executes on a Hewlett Packard (HP) 300 series workstation and uses HP VEEtest development software and the $\mathrm{C}$ programming language. Software tools were developed for data processing and analysis, and to provide a database organized by frequency bands, test runs, and sensors.

This paper describes the data acquisition system on board the aircraft and concentrates on the software portion. Hardware and software interfaces are illustrated and discussed. Particular attention is given to data acquisition and data format. The data reduction process is discussed in detail to provide insight into the characteristics, quality, and limitations of the data. An analysis of obstacles encountered during the data reduction process is presented.

\section{INTRODUCTION}

The goal of the FBL/PBW program is to provide advanced technology for future subsonic civil aircraft. This technology will include FBL/PBW systems and subsystems, modeling tools, and assessment techniques to aid in the prediction of the EME effects on flight critical electronics. The trend in commercial avionics is to integrate increasing numbers of digital computers into flight critical systems. In addition, the trend in aircraft construction is to use more composite materials, which may provide less radio frequency (RF) shielding than conventional metals. An increasing number of high power emitters will be operating in the future, putting more high powered radiation in the environment. Therefore, it is important for aircraft designers and developers to be equipped with EME modeling tools and assessment techniques that will enable them to understand the potential effects of radiation on aircraft electronics, design accordingly, and validate their designs.

In support of the FBL/PBW program, a series of flight tests were conducted to collect data while an aircraft is flying through an electromagnetic environment. The primary objective was to provide actual flight test data to be used in the validation of field levels predicted by EME computational and statistical modeling codes. In addition, the flight test data were to be available to the EM community for application to the development of other computational electromagnetic techniques and analyses. This paper describes the software for the data acquisition system used in the series of fly-by tests, and the methodology and software employed for the data reduction process. The flight test raw data were reduced to files containing columnar text that are organized by frequency, flight path, sensor, and time. The files contain the time of acquisition, the maximum levels detected by each sensor, and the aircraft position information at the time the field data were recorded. The data reside in a database that will be made available via a World Wide Web page. Of the original 70 Mbytes of raw data, 36 Mbytes of reduced data were produced. The original plan called for acquiring measurements in five different frequency bands, HF, VHF, UHF, S-Band, and $\mathrm{C}$-Band; however, due to weather and time constraints, no S-Band data were taken. Frequencies were chosen based on available transmitter sources at the NASA Wallops Flight Facility and the Voice of America, Greenville, North Carolina. Data were collected at the sites at frequencies of $25 \mathrm{MHz}, 173$ $\mathrm{MHz}, 430 \mathrm{MHz}$, and $5400 \mathrm{MHz}$. The HF and VHF data 


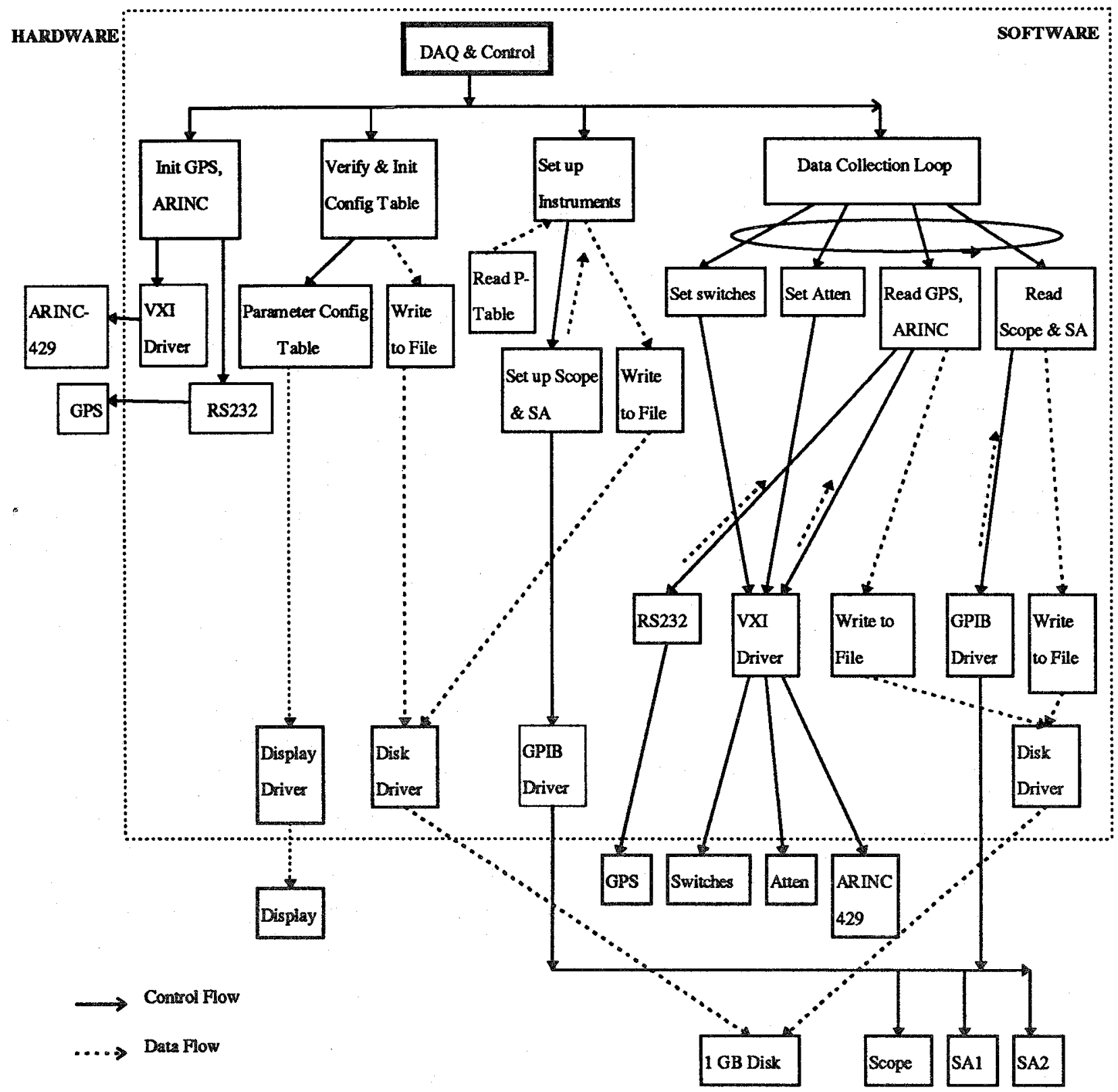

Figure 1. Control and Data Acquisition System Structure Chart

were collected from a source emitting a continuous wave (CW), and the UHF and C-Band source emitted a pulsed signal. A flight test plan [1] gives further details on the series of flight test experiments and locations.

\section{FLIGHT TEST EXPERIMENT}

\section{Configuration:}

The flight test data acquisition system was fully automated to allow for the acquisition of the greatest amount of information. Measurements of internal fields, external fields, and induced currents were acquired by multiplexing the sensors to the instruments by means of a switching matrix [1]. The data acquisition (DAQ) and control system structure are presented in Figure 1. The chart illustrates the interfaces between the software and hardware of the system, as well as the control and data flow. This discussion will concentrate only on the devices utilized by the data acquisition and control software.

The flight test instrumentation included a pair of HP8560E spectrum analyzers, later referenced as SA1 and SA2, and two channels of an HP54710 digital oscilloscope, later referenced as DS1 and DS2. An HP 
382 computer controller was used to control and access the measurement instruments via a General Purpose Interface Bus (GPIB). The aircraft ARINC 429 receiver and the global positioning system (GPS) receiver were accessed by the controller via a VXI bus and an R\$232 serial port. Sensors on the aircraft included VHF and UHF extemal antennas, a cabin long wire, a cabin DDot, an electronics bay D-Dot, a flight deck D-Dot, a cabin I-320, and an electronics bay I-320. The sensors were switched to the appropriate instruments in a predefined order. Figure 1 illustrates in some detail the various functions performed by the control software, including initialization, instrument set up, and continuous data acquisition. Details beyond the scope of this paper are provided in the flight test plan [1]. The test plan discusses the complete flight test system including instrumentation, sensors, switching matrix, attenuators, and amplifiers.

\section{Initialization and Setup:}

The software initialization functions included initializing processes to read from the ARINC 429 receiver and GPS receiver. The processes ran continuously, writing data into shared memory, where the main data acquisition software could retrieve the information and write it to disk. In addition, part of a configuration parameter table, presented in Figure 2, was initiated, verified, and recorded. The table contained instrument parameters and sensor switching order information. Elements of the configuration parameter table were used to set up the instruments and control the flow of data acquisition. The "Measurement Number" was used to determine data acquisition order, and the "Sensor" and "Instrument" fields were used to determine switching parameters. Input for controlling attenuation levels, trigger levels, and sensitivity levels were entered here. The pair of spectrum analyzers and the digital scope were set up by using the parameters given in Figure 3 . For the collection of data within the HF and VHF bands, the spectrum analyzer was considered to be more sensitive and therefore the primary measurement instrument. However, since the UHF and C-Band data were collected from a pulsed RF source, the digital scope was considered the primary instrument [2]. Characteristics of the digital scope allowed measurement of the time signature, which was needed to determine pulse rise time. Setting the spectrum analyzers to a zero span established an amplitude versus time mode. The result was that only the power levels at the frequency of interest were measured. After set up, the state of the digital scope was saved in the instrument's preamble and

$$
\begin{aligned}
& \text { Measurement Number }=1-1 \\
& \text { Sensor }=\mathrm{VHF} \\
& \text { Instrument }=\text { Scope-3 } \\
& \text { Attenuation }=0 \mathrm{~dB} \\
& \text { Sensitivity }=20 \mathrm{mV} / \mathrm{div} \\
& \text { Noise }=10 \mathrm{mV} \\
& \text { Trigger Level }=60 \mathrm{mV}
\end{aligned}
$$

Figure 2. A Portion of the Configuration Parameter Table

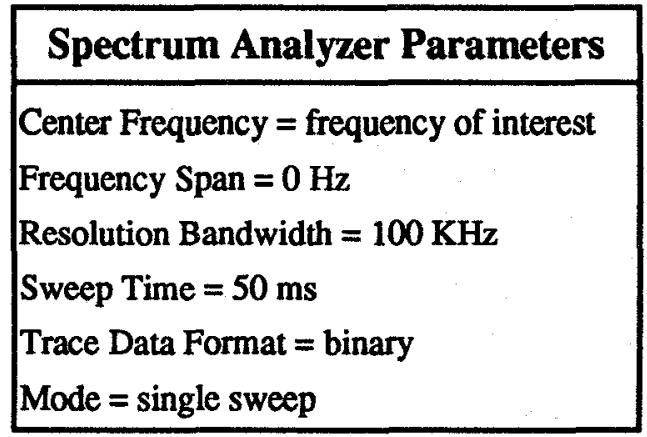

\begin{tabular}{|l|}
\hline \multicolumn{1}{|c|}{ Digital Scope Parameters } \\
\hline Timebase Reference $=$.2us (horizontal) \\
Vertical Scale $=50 \mathrm{mV} / \mathrm{div}$ \\
Sample Rate $=4 \mathrm{Ghz}$ \\
Trigger Level $=60 \mathrm{mV}$ \\
\\
\hline
\end{tabular}

Figure 3. Spectrum Analyzer and Digital Scope Setup Parameters.

then written to a file. The preamble included the scaling parameters, $x$ and $y$ origins, and $x$ and $y$ increments. The values were of particular importance to the data reduction computation that converted data from binary to scaled decimal format. During set up, DS2 was configured to trigger on DS1. If DS1 did not detect the assigned trigger level, it automatically triggered after 30 ms.

\section{Data Acquisition:}

As illustrated in Figure 1, data acquisition occurs continuously during each test run, looping until stopped. A test run is defined as a flight test that is linked to a 
specified flight path, date, and time. First, the sensors are switched to the appropriate instruments utilizing the switching matrix, and the attenuators are set to a level defined in the configuration parameter table. After a settling time, data are acquired from the GPS receiver, the ARINC receiver, SA1, SA2, DS1, and DS2. It requires approximately two seconds to cycle through all the sensors, both switching and acquiring data. That time increment is defined as an "epoch". Instrument data are written to files that are marked as either containing spectrum analyzer data or digital scope data. The results are spectrum analyzer data and digital scope data for each sensor. Each data file contains GPS and ARINC data, and two binary blocks of instrument data, waveform or spectrum. The acquisition process continues for an entire data run until stopped. The flight test plan [1] illustrates several different flight paths of differing lengths. The result is that the data for differing data runs varies in the number of epochs and in the number of data files generated. Forty nine of the 56 test runs are considered valid and complete.

\section{DATA REDUCTION}

\section{Design:}

The basic philosophy for the data reduction process was to verify the data at each reduction step by displaying it, analyzing it, and correlating it. By using this philosophy, the initial approach involved converting the binary data to scaled decimal format for each sensor and plotting waveforms and spectra to determine completeness, consistency, and validity of data. To accomplish this, display and analysis tools were developed in software. By using the display tools, the raw, transformed, and filtered DS waveforms were viewed. That allowed for a quick check of the data to determine missing or clipped data, and a relative comparison of sensor levels with respect to aircraft position.

Figure 4 presents the complete data reduction process, beginning with the raw data and ending with data plots and evaluations for each sensor. The process was developed and tested using the VHF data set. At each step in the process, it is possible to display the data for evaluation. To corroborate the accuracy of the measurements, the DS and SA data plots for a single sensor at a particular time are compared for similar patterns and levels. As the figure shows, the resulting VHF and HF portion of the database is generated from the SA data for each sensor. The process requires that the digital scope data be incorporated into the UHF and
C-Band portion of the database. From that database, plots are generated and examined for reasonableness and accuracy.

The objective of the reduction process is to convert the raw binary data recorded during each test run to a format usable for EME modeling and analysis code validation. The raw data are organized into $\mathrm{HF}, \mathrm{VHF}, \mathrm{UHF}$, and $\mathrm{C}$ band sets of data runs. The raw data contain SA and DS data files that are organized and mapped to particular sensors. During data collection, sensors are switched to specific instruments in a predetermined order as defined by the configuration parameter table. The data extracted from each raw data file during the data reduction process are mapped to the appropriate sensor. The result is reduced data organized by sensors for each test run.

\section{Data Reduction Tools:}

The data reduction process was initially defined using the VHF data set, since it is the largest, most complete set, and contains $\mathrm{CW}$ data. Minor changes to the process were applied to data of different frequencies. Software prototypes were written to explore methods for extracting, converting, correlating, plotting, and analyzing the data for each sensor. The process required constant observation and evaluation of the data to determine the best techniques for producing reliable and valid data. The prototypes were quickly developed to display and analyze data.

Software tools were created to display the raw time domain waveform, frequency domain, and filtered spectrum data from the two digital scope channels. The software can also display the aircraft position data and the portion of the configuration parameter table that applies to the related instrument data. A similar display can be applied to the spectrum analyzer data. The prototypes provide the basis for a data reduction tool set for analysis and reduction of the flight test data. All software was developed using HP VEEtest and the C language on an HP 382 workstation running the HP-UX operating system.

\section{Obstacles and Observations:}

During the development of the reduction process for the flight test data, analyses were performed to verify the accuracy of the instrument measurements. That involved comparing the DS data of a sensor with the SA data of the same sensor. The evaluation was initially performed using the VHF external antenna data from the VHF data set as more data were available for the sensor. The process followed that in Figure 4, "Digital Oscilloscope 
Digital Oscilloscope Sensor Data Reduction

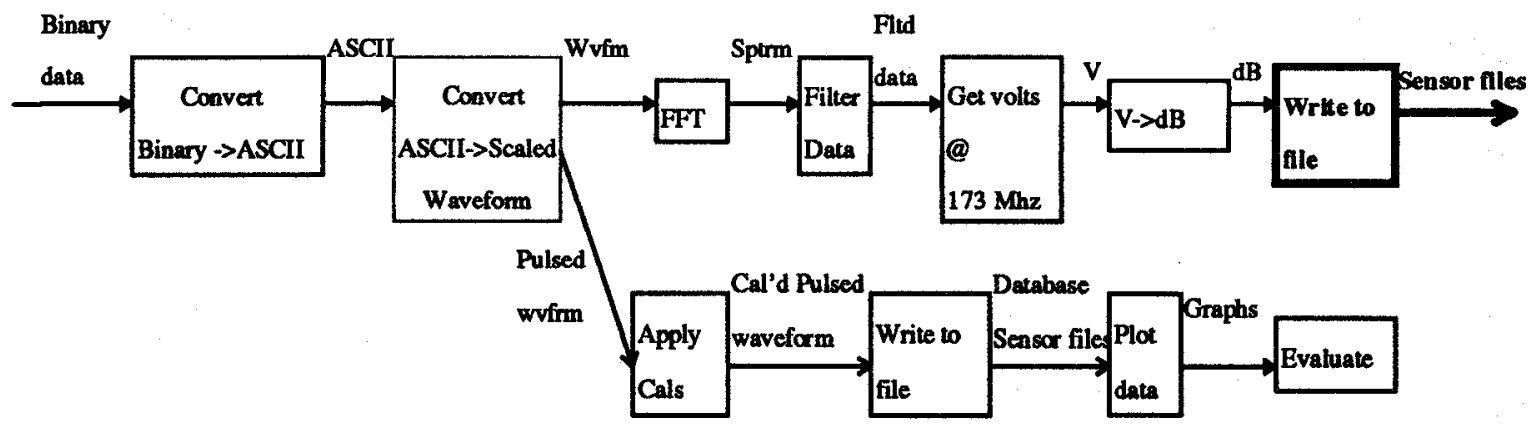

Spectrum Analyzer Sensor Data Reduction
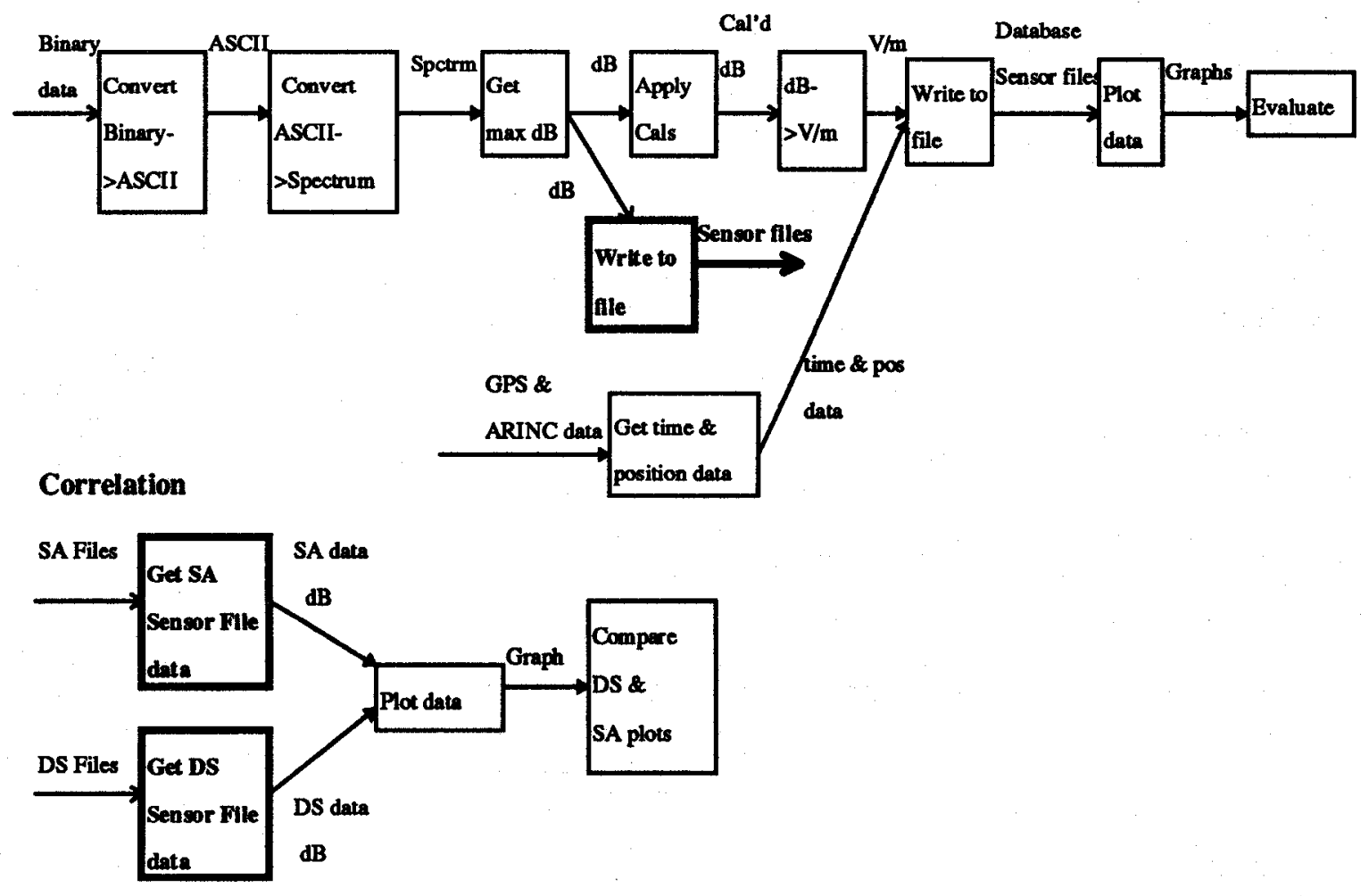

Figure 4: Data Reduction Process Data Flow Diagram

Sensor Data Reduction", where the DS data were transformed, filtered, and the amplitude level extracted at a frequency of $173 \mathrm{MHz}$. The process proceeded through the data for an entire test run, and wrote the values to a file. The DS data from the file were then plotted. The SA data for the same sensor and test run were reduced to extract and save the maximum values of the data to a file. The result was two data files, one containing DS values and the other SA values for one sensor across one test run. The SA data were then plotted against the DS data. The method was extended to include the other sensors in the data set.
While most of the DS and SA data plots produced favorable comparisons, there were instances where the DS measurements did not closely agree in magnitude with the SA measurements. Further investigation revealed that during actual flight test data acquisition, a command was sent to set the DS vertical scaling levels. Sometimes that resulted in changes to the levels. It is believed that measurements were acquired before the instrument completed executing the scale command. If the scaling level were changing, the data acquired has shown to be suspect. That event, however, only affects half of the DS VHF antenna measurements, the DS flight deck D-Dot 60 measurements, and the DS cabin D-Dot 60 measurements. Out of the seven different sensors read during a single VHF test run, only the DS 
measurements of two sensors and half of the DS measurements of another sensor are questionable. That analysis was also applied to the other test bands. The HF data set proved to be similar to the VHF data set. Due to time constraints, a study of the results of the UHF data set and C-Band data set is incomplete.

Since the primary measurement instrument for the HF and VHF bands was the spectrum analyzer [2], the data sets were reduced by using the acquired spectrum analyzer data and are included in the database. The reduced UHF and C-Band data sets will require additional study. Since the data sets contain pulsed data, and the DS was considered the primary instrument [2], further study and verification is considered fundamental. At this time, the database contains 41 test runs of reduced data acquired by the spectrum analyzer for the VHF and HF data sets. It is expected that additional data will be added as further evaluations are completed.

\section{Database Structure:}

Using the data reduction process as previously described, all sensor data collected via a spectrum analyzer from the HF and VHF frequency bands were processed and written to data files. The files were organized by frequency bands, test runs, and sensors. Included within each file in columnar text format and in sequential order were the measured levels, time, latitude, longitude, bearing, distance, and altitude of the aircraft for one test run. Data file names reflect the type of sensor data contained in the file:

$\begin{array}{ll}\text { vhf.dat } & \text { External VHF antenna } \\ \text { uhf.dat } & \text { External UHF antenna } \\ \text { clw.dat } & \text { Cabin long wire } \\ \text { fd60.dat } & \text { Flight deck D-Dot } 60 \\ \text { cd60.dat } & \text { Cabin D-Dot } 60 \\ \text { ed60.dat } & \text { Electronics bay D-Dot } 60 \\ \text { ci320.dat } & \text { Cabin I-320 } \\ \text { ei320.dat } & \text { Electronics bay I-320 }\end{array}$

\section{CONCLUSIONS}

The collection of EME data during the NASA B757 flight tests was completed in 1995 . The data acquisition and control software used during the test provided extensive automation of the process. A data reduction process was developed to reduce the flight data to a form that could be used to validate EME modeling predictions. The process was intended to be a rigorous application of reduction methods and analyses. The VHF data set was initially used to develop the reduction process, which was later extended to the other data sets. Observations during the reduction process revealed valid digital scope and spectrum analyzer measurements for both the VHF and HF data sets with the exception of the DS measurements from three of the sensors. The database was entirely populated with the sound data. No questionable data was included. The reduction of the VHF and HF data sets were completed and placed into a database that will soon be available. The UHF and CBand data have undergone part of the reduction process, but require further study.

\section{ACKNOWLEDGMENT}

The work described in this paper was performed under NASA contract NAS1-19000 at NASA Langley Research Center, Hampton, Virginia.

The author would like to acknowledge the guidance and contributions of both present and past team members. These members are Messrs. Kenneth Dudley, Charles Meissner, and Hal Carney, of NASA Langley Research Center, and Mr. Larry Corsa of Hewlett Packard. Also, credit is given to the NASA 757 crew members, the Aircraft Support Branch, and the Operations Engineering Branch for their support of the aircraft.

\section{REFERENCES}

[1] A. Poggio, Charles Meissner, "NASA FBL/PBW Program NASA Boeing 757 HIRF Test Plan Flight Tests", Ver. 1.0.5, December 8, 1995. [2] R. Zacharias, Carlos Avalle, Steve Pennock, A. Poggio, Robert Sharpe, "NASA FBL/PBW Program Boeing 737 HIRF Test Plan”, Revision 1, April 7, 1993. 